\title{
PERFIL OFTALMOLÓGICO DE PACIENTES INGRESSANTES NO SETOR DE GLAUCOMA DE UM SERVIÇO UNIVERSITÁRIO
}

\author{
OPHTHALMOLOGIC PROFILE OF PATIENTS GOING INTO THE GLAUCOMA WING OF AN UNIVERSITY SERVICE
}

\section{Ana Lúcia Gardim Demarco; ; Maria de Lourdes Veronese Rodrigues² \& Luís Antônio Demarco³}

\begin{abstract}
${ }^{1}$ Pós-graduanda (Doutorado). ${ }^{2}$ Docente. Departamento de Oftalmologia, Otorrinolaringologia e Cirurgia de Cabeça Pescoço. Faculdade de Medicina de Ribeirão Preto - USP. ${ }^{3}$ Oftalmologista.

CorrespondêncIA: Ana Lucia Gardim Demarco. Rua Cila, 3129 - CEP15090-430. São José do Rio Preto - SP - Tel : 0xx17-2341859. Email: lademarco@terra.com.br
\end{abstract}

DEMARCO ALG; RODRIGUES MLV \& DEMARCO LA. Perfil oftalmológico de pacientes ingressantes no Setor de Glaucoma de um serviço universitário. Medicina, Ribeirão Preto, 35: 478-486, out./dez. 2002.

RESUMO: A cegueira é um ônus muito grande para a sociedade e para o indivíduo em si. O Glaucoma é uma das maiores causas de cegueira no mundo. Por se tratar de uma doença insidiosa e assintomática em sua fase inicial, o seu diagnóstico é tardio e as perdas visuais são irreversíveis.

Os autores realizaram este trabalho com o intuito de traçar o perfil da demanda dos pacientes ingressantes no Setor de Glaucoma de um serviço universitário, para nortear metas para a prevenção da cegueira por glaucoma.

Foram estudados 100 pacientes portadores de hipertensão ocular e glaucoma crônico. Os exames mais relevantes foram a acuidade visual, alterações na cabeça do nervo óptico e campo visual.

Encontramos uma grande parcela de pacientes com, pelo menos, um dos olhos com visão menor ou igual a 20/200 ( 40,69\% dos pacientes com glaucoma), e outros pacientes com a mesma acuidade visual em ambos olhos (6,9\% dos pacientes com glaucoma). A relação escavação/disco maior ou igual a 0,6 foi encontrada em $53,4 \%$ dos olhos examinados, e assimetria entre as escavações, em 57\%. O campo visual apresentou alterações severas em 57,25\% dos olhos com glaucoma, devido à grande atrofia óptica glaucomatosa, em $26 \%$ dos olhos.

Concluímos que os pacientes com glaucoma chegam ao serviço médico, quando estão presentes grandes e irreversíveis alterações, limitando seu prognóstico. Portanto, medidas devem ser tomadas para minimizar esses números.

UNITERMOS: Glaucoma. Cegueira. Disco Optico.

\section{INTRODUÇÃO}

O glaucoma, entidade que se caracteriza por uma série de alterações morfofuncionais, pode ser acompanhado de grande variedade de manifestações clínicas. Definir glaucoma como sendo uma patologia causada pelo aumento da pressão ocular, seria simplificação generalizada de uma entidade complexa e até o momento parcialmente compreendida, pois não se sabe ainda qual o nível de pressão intra-ocular que poderá causar danos glaucomatosos ao olho, e não se conhece, por completo, o mecanismo que conduz à atrofia do nervo óptico. Sabe-se, contudo, que a lesão do nervo óptico está relacionada com perdas no campo visual e que a pressão ocular aumentada é o maior fator de risco $^{(1,2)}$. 
Na maioria dos casos, as alterações glaucomatosas progressivas, no nervo óptico e no campo visual, estão relacionadas com o aumento da pressão ocular, em alguns casos, no entanto, mesmo níveis normais de pressão ocular determinam lesão nas fibras axonais do nervo óptico, com perdas progressivas do campo visual $^{(1,2)}$.

Com isso, para que se estabeleça uma definição de glaucoma, deve-se levar em consideração a presença de 3 fatores associados: a pressão intra-ocular que nem sempre está elevada, alterações do nervo óptico e a perda progressiva do campo visual.

A prevalência do glaucoma, na população mundial, é relatada em trabalhos internacionais com valores que variam de 2 a $8 \%$, dependendo da região e da idade pesquisada ${ }^{(3 / 7)}$.

A população idosa é mais comumente atingida pelo glaucoma, mas pode ocorrer em outras faixas etárias, com consideráveis conseqüências econômicas, sociais e de saúde pública.

O glaucoma mais freqüente é o primário, também denominado glaucoma crônico de ângulo aberto ou glaucoma crônico simples, e é justamente essa a forma mais insidiosa da doença, cujos principais fatores de risco são : PIO elevada, hereditariedade, alterações do nervo óptico, idade avançada, raça negra, alta miopia e doenças vasculares ${ }^{(8,9)}$.

Acredita-se que o glaucoma possa ser responsável por cerca de $20 \%$ dos casos de cegueira em todo o mundo, sendo que, no Brasil, é uma entidade não menos significante e está entre as principais causas de cegueira ${ }^{(10 / 14)}$.

Sabe-se que a maioria dos pacientes glaucomatosos que chegam aos serviços oftalmológicos já apresentam perdas campimétricas. Contudo, não se sabe qual a magnitude do problema no Brasil.

O presente estudo foi delineado para verificar o perfil das condições oculares de pacientes encaminhados para tratamento especializado de glaucoma, com os seguintes objetivos específicos:

1. quantificar a acuidade visual central, com a melhor correção óptica possível;

2. medir as pressões intra-oculares na primeira consulta;

3. quantificar e documentar a relação escavação/disco óptico;

4. verificar a presença e forma de alterações campimétricas;

5. classificar os glaucomas, principalmente, pela gonioscopia.

\section{MATERIAL E MÉTODOS}

Este estudo foi feito com 100 pacientes que ingressaram no Setor de Glaucoma do Hospital de Base de São José do Rio Preto, Estado de São Paulo, no período de abril de 1994 a setembro de 1996, após o delineamento do projeto de pesquisa.

Os critérios de inclusão para a participação no estudo foram a PIO maior ou igual a $21 \mathrm{mmHg}$, na primeira consulta, em, pelo menos, um dos olhos, em pacientes sem antecedentes para cirurgia glaucomatosa ou uso de colírio antiglaucomatoso, e em pacientes com história de cirurgia antiglaucomatosa prévia ou, que, na ocasião da consulta, estivessem usando medicação antiglaucomatosa, estes pacientes foram considerados para estudo independente da PIO. Foram excluídos do estudo os pacientes com glaucoma congênito, agudo e secundário. Todos os exames foram realizados pelos autores.

Participaram do estudo 42 homens e 58 mulheres, sendo que 41 procediam da cidade de São José do Rio Preto e 59, de outras regiões do Estado de São Paulo.

Para cada paciente foi preenchida uma ficha com nome completo, número de registro no hospital, sexo, data de nascimento, idade, cor da pele, procedência e tratamentos antiglaucomatosos prévios e em uso.

Os exames realizados foram: refração dinâmica e medida da acuidade visual pela tabela de Snellen, a 6 metros, tonometria de aplanação, biomicroscopia do segmento anterior e de fundo e perimetria computadorizada. Foi, também, realizada retinografia em todos os pacientes em que foi possível a visualização do fundo de olho, para a documentação das condições da cabeça do nervo óptico.

A técnica de gonioscopia utilizada foi a indireta, após a instilação de colírio anestésico, com lente tipo Goldmann, de um espelho com inclinação de $62^{\circ}$. A configuração do ângulo da câmara anterior foi dividida entre ângulo aberto ou estreito, sem levar em consideração a curvatura periférica da íris, a graduação do ângulo ou o ponto de inserção iriano, considerando-se a amplitude do mesmo.

Os pacientes foram divididos em hipertensos oculares, quando não tinham alteração no campo visual, e com glaucoma crônico de ângulo aberto ou estreito, quando apresentavam alterações campimétricas, características de glaucoma.

A avaliação da cabeça do nervo óptico foi feita pela biomicroscopia indireta à lâmpada de fenda, modelo 
Topcon SL-2E e lente de 90 dioptrias. A maior relação eixo de escavação/tamanho da cabeça do disco óptico foi anotada na forma decimal.

A documentação fotográfica do disco óptico foi feita para todos os pacientes, e foi possível a visualização do fundo do olho, por meio de retinografia cencentrada no disco óptico, usando-se o Retinógrafo Topcon TRC 50X e filme Kodacrome, ASA 100.

A campimetria computadorizada foi realizada com a melhor correção óptica possível, inclusive a graduação cilíndrica e com diâmetro pupilar maior que 3 $\mathrm{mm}$, sempre que possível. O aparelho utilizado foi o campímetro automatizado, OCTOPUS 123, versão 9.09, com peridata e iluminação de fundo de $31 \mathrm{asb}$. O programa de escolha, para a maioria dos pacientes, foi o G1X.

O programa G1X testa 59 pontos dentro dos 30 graus de fixação. O estímulo usado é o de tamanho III de Goldmann, com um tempo de exposição de 100 ms. Os pontos estão distribuídos de forma mais agrupada na região mais central, visando à melhor pesquisa dos escotomas paracentrais, freqüentes no glaucoma ${ }^{(15)}$.

Para os pacientes com baixa visão, quando não foi possível a avaliação campimétrica com o programa G1X, foi usado o programa Custon Test, no qual se usa o estímulo $\mathrm{V}$ de Goldmann, com uma exposição de $200 \mathrm{~ms}$, testando 64 pontos nos 30 graus centrais. A estratégia de exame é a apresentação de um estímulo luminoso de intensidade máxima. Se a primeira resposta do paciente é positiva, inicia-se a detecção de sensibilidade à luz através da estratégia normal de duplo cruzamento, descrita acima. Se a primeira resposta do paciente é negativa, o limiar, nesse ponto, é considerado como zero decibel, e aí não haverá repetição de teste.

Os defeitos campimétricos foram definidos conforme os critérios de Caprioli, 1992 ${ }^{(16)}$, onde o escotoma e o degrau nasal se baseiam no encontro de dois ou mais pontos contíguos com $10 \mathrm{~dB}$.

Os achados do campo visual foram agrupados em:

0 sem alteração no campo visual;

1 presença de escotomas paracentrais;

2 presença de escotomas paracentrais e escotoma arqueado e/ou degrau nasal;

3 presença de escotomas paracentrais e escotoma arqueado e/ou degrau nasal e lesão no campo visual, no outro hemicampo;

4 campo visual tubular ( $<20$ graus $)$;

5 remanescente temporal de visão;

6. campo visual inviável.

\section{RESULTADOS}

A distribuição dos pacientes, segundo a cor da pele, foi constituída de $72 \%$, branca, $23 \%$, parda e $5 \%$, negra.

A faixa etária predominante foi de 61-70 anos e $78 \%$ dos pacientes tinham 51 anos ou mais.

Muitos pacientes já ingressaram, no Setor de Glaucoma do Hospital do Base de São José do Rio Preto, com acuidade visual bastante comprometida, em, pelo menos, um dos olhos. No entanto, a maioria dos olhos (135 olhos - 67,5\%) apresentavam acuidade visuais na faixa de 20/20 a 20/60.

Dos pacientes estudados, 40 sabiam que tinham glaucoma, com diagnóstico feito em diferentes épocas, em outros serviços. No Hospital de Base de São José do Rio Preto, foi feito o diagnóstico dos outros 60 pacientes.

Na primeira consulta, os pacientes apresentavam PIO entre 8 e $55 \mathrm{mmHg}$ (mediana de $23 \mathrm{mmHg}$ ). O gráfico 1 mostra a distribuição da pressão ocular em 198 olhos estudados; outros dois olhos atróficos inviabilizaram a medida da pressão ocular.

A distribuição da relação escavação/disco (E/ D) óptico, nos 200 olhos estudados, está no Gráfico 2.

A documentação fotográfica (retinografia) foi feita em todos os olhos que apresentavam condições oculares para o exame. As causas que inviabilizaram a avaliação e a documentação da relação escavação/ disco óptico, em 12 olhos, foram: atrofia ocular (2 olhos), catarata (8 olhos), descolamento de retina (2 olhos).

A assimetria na relação escavação/disco óptico foi maior ou igual a 0,1 , entre os dois olhos, em $57 \%$ dos pacientes participantes.

0s achados do campo visual foram agrupados em 7 categorias. A distribuição dos resultados está expressa no Gráfico 3.

Com base na presença de alteração campimétrica, característica de glaucoma, os pacientes foram categorizados em hipertensos oculares (14\%) e pacientes com glaucoma $86 \%$. Por sua vez, com base na gonioscopia, os pacientes com glaucoma crônico foram subdivididos em ângulo aberto ou estreito, sendo que 65 pacientes apresentaram glaucoma de ângulo aberto.

\section{DISCUSSÃO}

A Organização Mundial de Saúde (OMS) sugere que $80 \%$ da cegueira encontrada nos países em 


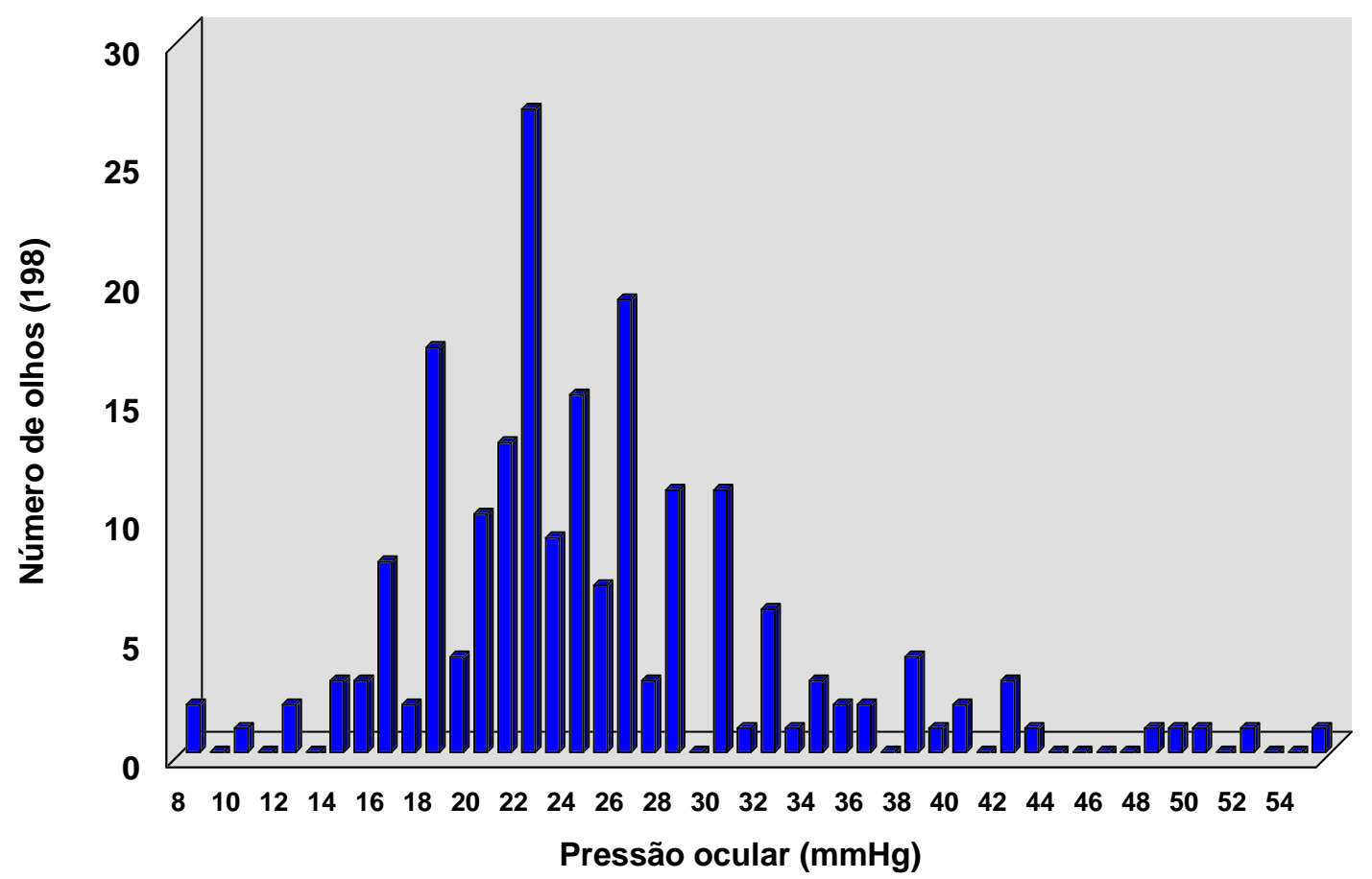

Gráfico 1- Distribuição da PIO nos 198 olhos avaliados, na ocasião do ingresso dos 100 pacientes que participaram do estudo, na primeira consulta realizada no Setor de Glaucoma do Hospital de Base de São José do Rio Preto-SP.

desenvolvimento pode ser prevenida ou curada. Em 1990, a OMS estimou em 35 milhões o número de cegos na população mundial (acuidade visual no melhor olho menor, com melhor correção ou igual a 0,05 ). Esse número aumentaria aproximadamente para 50 milhões, se o nível de acuidade visual considerado fosse igual ou inferior a $0,1^{(17)}$.

Segundo Temporini, 1991(18), os Programas de Oftalmologia em Saúde Pública devem priorizar ações relacionadas a: prevenção de estados conducentes à cegueira e à incapacidade visual, promoção da saúde ocular e reabilitação dos deficientes visuais cegos. Para o planejamento dessas ações se faz necessário o conhecimento objetivo da realidade a qual se destinam. A pesquisa científica permite a obtenção desse conhecimento, fornecendo subsídios para o estabelecimento de linhas de ação compatíveis com a realidade.

Existem controvérsias quanto aos níveis de PIO, considerados normais. Leydhecher, $1964{ }^{(19)}$ realizou um estudo epidemiológico na Alemanha e considerou $2 \mathrm{lmmHg}$ como nível superior da normalidade. Estudos realizados na América do Sul e no Brasil, em particular, mostraram que as médias das PIO de populações sul-americanas são menores. Por exemplo, Lauretti Filho \& Romão, $1966^{(20)}$ e Lauretti Filho, 1972
(21) estudaram dois grupos de pacientes na cidade de Ribeirão Preto - SP, encontrando médias de PIO por volta de $13 \mathrm{mmHg}$. No que diz respeito ao limite superior da normalidade, os valores encontrados foram, respectivamente, 20,83 no primeiro trabalho e 19,47 no segundo (do qual participaram pessoas menores de 40 anos), ou seja, aproximadamente 21 e $20 \mathrm{mmHg}$. Calixto, $1967^{(22)}$ estudou, em Belo Horizonte-MG, diferentes grupos etários e encontrou média de 12,87 $\mathrm{mmHg}$ e, como limite superior da normalidade, 19,53 $\mathrm{mmHg}$.

O fato de terem participado do estudo não só pessoas que residiam na cidade de São José do Rio Preto, mas, também, pacientes oriundos de outras regiões do Estado de São Paulo, é explicado pelo fato de que o Hospital de Base ser um centro de referência.

Pensamos que a distribuição da cor da pele, em nossa amostra, segue a distribuição da população da região, para as cores branca, parda e negra. No entanto, não está representada a cor amarela. Supõe-se que seja devido à pequena distribuição dos mesmos na região e devido a fatores socioeconômicos e culturais, que fazem com que essas pessoas procurem menos os serviços públicos. 


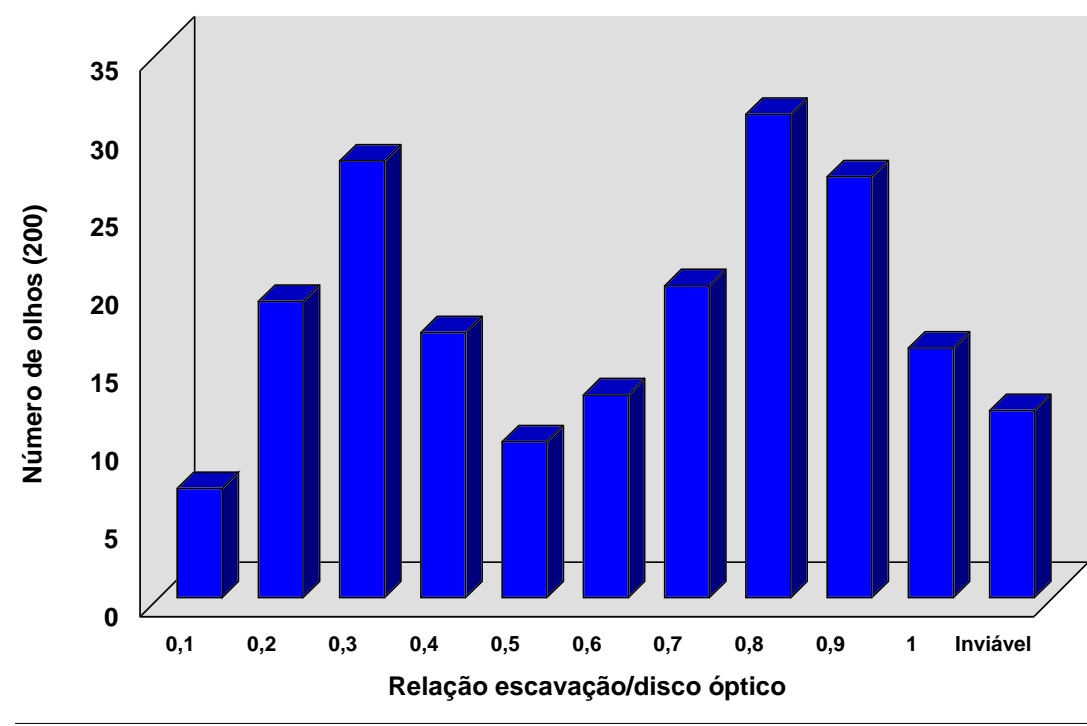

Grafico 2 - Distribuição da relação escavação/disco óptico nos 200 olhos avaliados, na ocasião da primeira consulta realizada no Setor de Glaucoma do Hospital de Base de São José do Rio Preto,SP

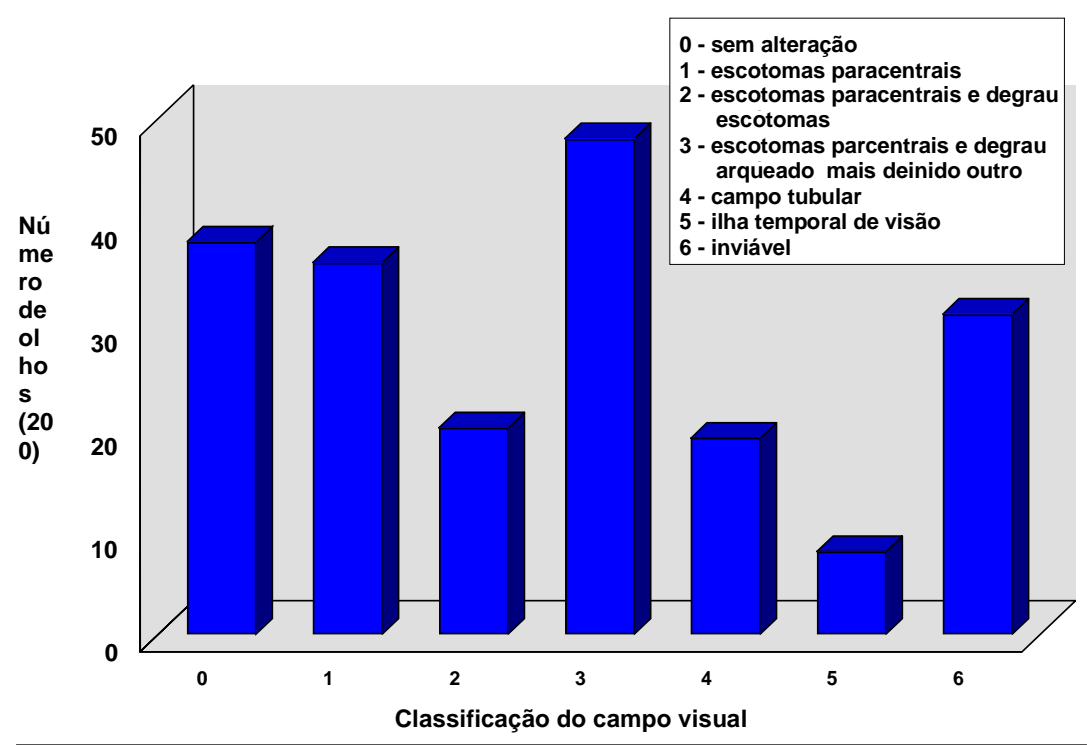

Gráfico 3 - Distribuição dos resultados da perimetria computadorizada, realizada em 169 olhos dos 100 pacientes, na ocasião do ingresso no Setor de Glaucoma do Hospital de Base de São José do Rio Preto,SP.

Quando avaliamos a distribuição por sexo, percebemos a predominância do sexo feminino em $58 \%$ dos pacientes avaliados, dado semelhante encontrado por Silva, Rodrigues \& Lauretti, $1998^{(23)}$. Sabemos que o glaucoma crônico simples afeta, de forma semelhante, ambos os sexos, porém o glaucoma crônico de ângulo estreito é mais freqüente no sexo feminino $(24,25,26)$ e aparece, em nosso estudo, em 21 pacientes, sendo 17 pacientes do sexo feminino, justificando nossa amostra.
Encontramos, em nossa amostragem, 14 pacientes hipertensos oculares, 65 com glaucoma crônico de ângulo aberto e 21 com glaucoma crônico de ângulo estreito. Consideramos o fator decisivo para a classificação dos hipertensos oculares e glaucomatosos a presença ou não de perdas campimétricas.

Dos 14 pacientes hipertensos oculares, 3 vieram de outros serviços, com tratamento prévio para glaucoma. Acreditamos que, fora de serviços universitários, esse número seja maior, pois a dificuldade na realização do campo visual é grande, sendo o parâmetro adotado para tratamento ou não do glaucoma, baseado exclusivamente na PIO.

Como era esperado, predominaram os pacientes de faixas etárias mais elevadas, também encontradas na literatura ${ }^{(27,28,29)}$. As faixas etárias predominantes foram as de 61 a 70 anos, com 37 pacientes, seguidas pela faixa etária de 51 a 60 anos, com 27 pacientes.

A acuidade visual foi melhor ou igual a 20/60, em 135 olhos. Encontramos 35 pacientes $(40,69 \%$ dos pacientes com glaucoma) com um dos olhos com acuidade visual menor ou igual a 20/200 e porcentagem semelhante $(36,12 \%)$ foi encontrada na literatura ${ }^{(28)}$. Seis pacientes $(6,9 \%$ dos pacientes com glaucoma) apresentavam acuidade visual menor ou igual a 20/200 nos dois olhos, sendo considerados cegos legalmente. Acreditamos que a quantidade de cegos por glaucoma seja maior, pois se trata de uma doença assintomática.

$\mathrm{Na}$ maioria dos pacientes, o diagnóstico foi realizado em nosso serviço (60\%). Dos pacientes que vieram encaminhados de outros serviços, $18(22,5 \%)$ já haviam sido submetidos a algum tipo de cirurgia antiglaucomatosa, $10(12,5 \%)$ estavam usando medicação oral: acetazolamida e 75 (93,75\%) usavam, pelo menos, uma medicação tópica. As cirurgias prévias e o uso de medicação, na primeira medida da pressão ocular, justifica a distribuição dos valores encontrados. 
As medicações tópicas referidas, como tratamento por ocasião da primeira consulta no Serviço de Glaucoma, foram os agentes colinérgicos (Piocarpina ${ }^{\circledR}$ ), agentes adrenérgicos (Propine ${ }^{\circledR}$ ) e os betabloqueadores não seletivos (Timolol®). Nessa ocasião, ainda não estavam disponíveis, no mercado, as novas drogas antiglaucomatosas, como os betabloqueadores seletivos, os inibidores tópicos da anidrase carbônica, os agonistas adrenérgicos e as prostaglandinas.

A grande maioria dos pacientes, que nos foi encaminhada, nunca tinha sido submetida à avaliação do campo visual. Esse fato mostra a enorme dificuldade encontrada para o real diagnóstico do glaucoma fora de grandes centros e a falta de possibilidade de um bom acompanhamento.

Ao avaliar a cabeça do nervo óptico, encontramos diversidade de padrões, o que, provavelmente, represente diferentes populações de pacientes com glaucoma, possivelmente com diferentes mecanismos patológicos, também descritas por Nicolela \& Drance, $1996^{(30)}$. Estudos referem que a relação escavação/disco óptico é mais sensível para propósitos de screening do glaucoma, porém a avaliação da rima neural é mais sensível no acompanhamento dos pacientes ${ }^{(31)}$.

Para este estudo, avaliamos a relação escavação/disco óptico em seu maior eixo, pela da observação da cabeça do nervo óptico, em lâmpada de fenda e pela fotodocumentação do mesmo. Os autores acreditam que essa rotina de avaliação seja tecnicamente fácil de execução e não tenha custos tão elevados, podendo ser realizada rotineiramente, nos pacientes com hipertensão ocular e glaucoma, por oftalmologistas generalistas, em seus consultórios privados ou em serviços universitários, onde haja uma rotatividade muito grande dos médicos que atendem os pacientes. Por mais minucioso que o oftalmologista seja, em desenhar ou descrever as nuances da cabeça do nervo óptico, a fotodocumentação é menos sujeita a descrições subjetivas, facilitando o acompanhamento das alterações na escavação e na rima neural do nervo óptico dos pacientes ${ }^{(32)}$.

Em nossos pacientes, ao avaliarmos a relação escavação/disco óptico, encontramos 107 olhos $(53,4 \%)$ com escavação maior ou igual a 0,6 . São raras as escavações acima de 0,65 em indivíduos normais, sendo encontradas em apenas $5 \%$ dos casos (33). Em 12 olhos, não foi possível a observação da cabeça do nervo óptico.

A assimetria, no tamanho da escavação, é outro achado que parece estar estreitamente associado à presença de glaucoma. Em indivíduos normais, a incidência de assimetria maior que 0,1 parece girar em torno de $8 \%$ e maior que 0,2 , em apenas $0,5 \%$ dos casos ${ }^{(33)}$. Em nosso estudo, encontramos uma assimetria maior que $0,1 \mathrm{em} 57 \%$ dos pacientes e assimetria maior que $0,2 \mathrm{em} 30 \%$.

Alguns pacientes apresentaram outras alterações na cabeça do nervo óptico, descritas por Susanna, $1996^{(34)}$ como hemorragia, palidez do anel neurorretiniano, anel peripapilar de Primrose, notch, sinal da baioneta e fosseta adquirida.

As alterações anatômicas, no disco óptico, mais típicas de glaucoma são: a progressiva escavação do disco óptico, afinamento de rima neurorretiniana e danos na camada de fibras nervosas. (Fig 1) Do ponto de vista funcional, os defeitos no campo visual, apresentam-se na área paracentral e periférica e se tornam, gradualmente, mais óbvios até, finalmente, afetarem a área de fixação e reduzirem a acuidade visual. A relação entre os defeitos funcionais e anatômicos tem sido objeto de muitos estudos ${ }^{(35,36)}$. A medida do campo visual é o teste de função visual, mais comumente usado para avaliar o progresso da doença e estabelecer um tratamento adequado.

Ao avaliarmos as condições do campo visual dos pacientes do estudo, encontramos uma grande porcentagem de olhos com perdas campimétricas severas, como a presença de alteração nos dois hemicampos, visão tubular e remanescente temporal de visão. Nesse grupo, estão 75 olhos, que equivalem a 57,25\% dos olhos glaucomatosos, onde foi possível a realização do exame.

A utilização do estímulo de tamanho $\mathrm{V}$, na perimetria computadorizada, mostrou-se eficiente na avaliação campimétrica de pacientes com baixa visão, encontrada nos estágios avançados da atrofia óptica glaucomatosa. Seria impossível mensurar o campo visual desses pacientes com estímulos de tamanho III. Na literatura, há outros autores que tiveram o mesmo sucesso ${ }^{(37,38)}$.

Dos 31 olhos, onde não foi possível a realização do campo visual, $26(83,87 \%)$ tinham uma atrofia óptica glaucomatosa em fase final.

Ao fazermos o cruzamento de dados entre a relação escavação/disco óptico e campo visual, observamos que $84,21 \%$ dos olhos, com relações escavação/disco óptico menores ou iguais a 0,5 , não apresentavam alterações perimétricas e 89,4\% dos olhos, com relação escavação/disco óptico maior ou igual a 0,6 , apresentaram alterações perimétricas. 


\section{CONCLUSÕES}

O presente trabalho comprovou que o acesso aos serviços universitários, muitas vezes, ocorre numa fase avançada do glaucoma.

Um importante fator agravante, na problemática da cegueira por glaucoma, é a não adesão ao tratamento. Como se trata de uma doença praticamente assintomática e crônica, a falta de conscientização leva, muitas vezes, à irregularidade ou abandono do tratamento $^{(39 / 44)}$.

É um consenso que o screening para o glaucoma não deva ser baseado em apenas um parâmetro, como a pressão ocular ${ }^{(45,46,47)}$. A informação sobre a aparência da cabeça do nervo óptico e a avaliação funcional da visão são de grande importância.

Vários estudos apontam que, quando se faz um planejamento para a saúde pública, é mais barato despender gastos com a prevenção ${ }^{(48,49,50)}$. Nos países em desenvolvimento, os escassos recursos destinados à área de saúde devem atender prioridades múltiplas e distintas, nem sempre privilegiando programas de tônica preventiva ${ }^{(51)}$.
Para que se possa prevenir a cegueira por glaucoma, os autores propõem:

- o encontro de meios para melhorar o acesso da população aos serviços médicos, através da implantação de serviços de Oftalmologia em cidades da região, reduzindo a demanda aos hospitais universitários, que deveriam ser, na realidade, responsáveis pelo atendimento terciário;

- a elevação do nível de atendimento oftalmológico nos Postos de Saúde, equipando-os, para que possam ser realizadas consultas oftalmológicas básicas, com tonometria e fundoscopia;

- a promoção de campanhas em grandes centros, a fim de alertar e educar a população a respeito do glaucoma e, eventualmente, descobrir casos novos;

- que cada consulta para troca de óculos seja um meio para a conscientização do paciente a respeito da necessidade do exame oftalmológico periódico, não apenas visando à troca da graduação dos mesmos, mas, principalmente, à prevenção das patologias oculares.

DEMARCO ALG; RODRIGUES MLV \& DEMARCO LA. Ophthalmologic profile of patients going into the glaucoma wing of an university service. Medicina, Ribeirão Preto, 35: 478-486, oct./dec.2002.

ABSTRACT: Blindness is a very big onus on society and on the person itself. The glaucoma is one of the biggest causes of blindness in the word. Being an insidious and assymptomatic disease at the onset, its diagnosis is late and the visual losses are irreversible.

Having this preocupation, the author accomplished this study to draw the profile of the patients entering the Glaucoma Sector of an University Hospital in order to identify goals to prevent blindness caused by glaucoma.

One hundred patients with ocular hypertension and chronic glaucoma were studied. The most relevant exams were the visual acuity, alterations in the optic nerve head and visual field.

We found a great number of pacients having at least one of the eyes with vision less or equal to $20 / 200(40.69 \%$ of the pacients with glaucoma) and other pacients having the same visual acuity in both eyes $(6.9 \%$ of the eyes with glaucoma). The relation excavation/optic disc bigger or equal to 0,6 was found in $53.4 \%$ of the examined eyes and in $57 \%$ of them was found asymmetry among the excavation. The visual field showed severe alterations in $57.25 \%$ of the eyes with glaucoma, so that it is due to the big glaucornatous optic atrophy in $26 \%$ of the eyes.

It was observed that patients with glaucoma go to the medical service when major and irreversible alterations are present, limiting its prognosis. Therefore, efforts must be taken in order to minimize such numbers.

UNITERMS: Glaucoma. Blindness. Optic Disc. 


\section{REFERÊNCIAS BIBLIOGRÁFICAS}

1 - O'BRIEN C; SCHWARTZ B; TAKENORI T \& WU DC. Intraocular pressure and the rate of visual field loss in chronic openangle glaucoma. Am J Ophthalmol 111: 49I-500, 1991.

2 - ODBERG T. Visual field prognosis in early glaucoma. Acta Ophthalmol 71: 72I-726, 1993.

3 - MASSON RP; KOSOKO O; WILSON MR; MARTONE J.F; COWAN IR CL; GEAR JC \& ROSS-DEGNAN D. National survey of the prevalence and risk factors of glaucoma in St. Lucia, West Indies. Ophthalmology 96: 1363-1368, 1989.

4 - KLEIN BEK; KLEIN R; SPONSEL WE; FRANKE T; CANTOR LB; MARTONE J \& MENAGE MJ. Prevalence of glaucoma - The Beaver Dam Eye Study. Ophthalmology 99:1499-1504, 1992

5 - LESKE MC; CONNELL MAS; SCHACHAT AP \& HYMAN L - The Barbados eye study. Arch Ophthalmol 112: 821-829, 1994.

6 - MITCHELL P; SMITH W; ATTEBO K \& HEALEY PR. Prevalence of open-angle glaucoma in Australia. The Blue Mountains eye study. Ophthalmology 103: 1661-1669, 1996.

7 - BONOMI L; MARCLINI G; MARRAFA M; BERNARDI P; DE FRANCO I; PERFETTI S; VAROTTO A \& TENNA V. Prevelence of glaucoma and intraocular pressure distribution in a definid population - The Egna-Neumarkt study. Ophthalmalogy 105: 209-2|4, 1998.

8 - LESKE MC. The epidemiology of open-angle glaucoma: A review. Am J Epidemiol 118: 166-191, 1983.

9 - LAURETTI FILHO A \& LAURETTI CR. Glaucomas do adulto. In: RODRIGUES MLV. Oftalmologia clínica. Cultura Médica Rio de Janeiro, cap.16, p.243-278, 1992.

10 - QUIGLEY HA. Number of people with glaucoma wordwide. Br J Ophthalmol 80: 1389-1393, 1996

11 - SILVA MRBM; SCHELLINI AS; KAMEGASAWA A; HEIMBECK FJ \& CARANDINA L. Levantamento de cegueira em Botucatu - prevalência e causas. Rev Bras Oftalmol 45: 18-23, 1986

12 - MOREIRA ATR; MOREIRA JR. CA \& ARANA J. Causas de cegueira no Instituto de cegos do Paraná. Arq Bras Oftalmol 54: 275-278, 1991

13 -LEAL DB; TAVARES SS; VENTURA LO \& DANTAS H. Prevalência das causas de cegueira no Instituto dos cegos de Pernambuco. Rev Bras Oftalmol 54: 49-52, 1995.

14 - FREITAS JAH; SILVA HMB; BERTOTTI GSA \& COSTA IR. Incidência de cegueira no primeiro atendimento ambulatorial do Hospital Universitário - PUCCAMP. Rev Bras Oftalmol 56: 19-25, 1997

15 - INTERZEAG. Medical technology - Octopus Perimeter 123. Catálogo 06192. Interzeag, Northboro, MA, 1992. 49p.

16 - CAPRIOLI J. Discrimination between normal and glaucomatous eyes. Invest Ophthalmol Vis Sci 33: 153-159, 1992.

17 - COSTA VP; ALMEIDA GV \& KARA-JOSÉ N. Prevenção da cegueira por glaucoma. Arq Bras Oftalmol 61: 1356-1360, 1998.
18 - TEMPORINI ER. Pesquisa de oftalmologia em Saúde Pública: Considerações metodológicas sobre fatores humanos. Arq Bras Oftalmol 5: 279-28I, 1991

19 - LEYDHECHER W. El glaucoma en la pratica. Barcelona, Ediciones Tornay, 1964, I30p

20 - LAURETTI FILHO A; ROMÂO E. Estudo comparativo entre tonometria de aplanação e tonometria de impressão. Rev Bras Oftalmol 25: 29-36, 1966.

21 - LAURETTI FIlho A. Contribuição ao estudo da chamada "reação oftalmotônica consensual" na tonografia. Tese de Livre Docência, Faculdade de Medicina de Ribeirão Preto da USP, Ribeirão Preto, 1972.

22 - CALIXTO N. Pressão ocular, curva diária de pressão, rigidez parietal, coeficientes tonográficos (medidas de normalidade em diferentes grupos etários). Tese de Livre Docência, Faculdade de Medicina da UFMG, Belo Horizonte, 1967.

23 - SILVA, FLM; RODRIGUES, MLV \& LAURETTI,CR. Condições Oftalmologicas no primeiro atendimento de pacientes com cegueira por glaucoma. Trabalho apresentado. XIII Congresso Brasileiro da Prevenção da Cegueira e Reabilitação Visual, 7 a 10 de setembro de 1998, Rio de Janeiro.

24- CONGDON N; WANG F \& TIELSCH JM. Insusues in the epidemiology and population based screening of primary angle-closure glaucoma. Surv Ophthalmol 36:411-423, 1992.

25 - SALMON JF; MERMOUD A; IVEY A; SWANEVELDDER AS \& HOFFMAN N. The prevalence of primary closure glaucoma and open angle glaucoma in Mamre, Western Cape, South Africa. Arch Ophthalmol 111: 1263-1269, 1993.

26 - LAURETTI CR; RODRIGUES MLV \& LAURETTI FILHO A. Epidemiologia do glaucoma. In: DIAS JFP. Glaucoma. Cultura Médica, Rio de Janeiro, cap.1, p.1-5, 1998.

27 - GULLO RM; COSTA VP; BERNARDI L \& KARA-JOSÉ N Condições visuais de pacientes glaucomatosos em um hospital universitário. Arq Bras Oftalmol 58: 189-192, 1996.

28 - RODRIGUES AC; SILVA MRBM \& SCHELLINI AS. Número de olhos cegos por glaucoma detectados em primeira consulta num hospital universitário. Arq Bras Oftalmol 61: 572 578,1998

29 - RODRIGUES MLV; LAURETTI CR \& LAURETTI FILHO A Prevenção da cegueira por glaucoma. In: DIAS JFP. Glaucoma. Cultura Médica, Rio de Janeiro, cap.2, p.6-9, 1998.

30 - NICOLELA MT; DRANCE SM. Various optic nerve appearances - clinical correlations. Ophthalmology 103: 640-649, 1996.

31 - DAMMS T \& DANNHEIM F. Sensitivity and specificity ofoptic disc parameters in chronic glaucoma. Invest Ophthalmol Vis Sci 34: 2246-2250, 1993.

32 - KLEIN BEK; MOSS SE; MAGLI YL; KLEIN R; JOHNSON JC; ROTH H. Optic disc cupping as clinicaly estimated from photographs. Ophthalmology 94: 1481-1483, 1987. 
33 - ALMEIDA HG; FIGUEIREDO CL \& SAFADY M. Topografia da papila e análise da camada de fibras nervosas. In: DIAS JFP. Glaucoma. Cultura médica, Rio de Janeiro, cap.7, p.4860, 1998.

34 - SUSANNA JR R. Nervo óptico no glaucoma: Aspectos oftalmoscópicos. EDUSP, São Paulo, 1996. 94p

35 - WEBER J; DANNHEIM F\& DANNHEIM D. The topographical relationship between optic disc and visual field in glaucoma. Acta Ophthalmol 68: 568-574, 1990.

36 - JAY JL \& MURDOCH JR. The rate of visual field loss in untreated primary open angle glaucoma. $\mathbf{B r} \mathbf{J}$ Ophthalmol 77: 176-178, 1993.

37 - ZALTA AH. Use the central $10^{\circ}$ field and size $\mathrm{V}$ to evaluate and monitor small central island of vision in end stage glaucoma. Br J Ophthalmol 75: 151-154, 1991

38 - COMEGNO PEC; COSTA VT; SILVA SARF \& KARA-JOSÉ N. Perimetria computadorizada em pacientes glaucomatosos com visão subnormal. Arq Bras Oftalmol 58: 346-353, 1995.

39 - KARA-JOSÉ N; PEREIRA VL; MELO HFR; URVANEJA AJ \& BRASIL JR W. Criação do núcleo de prevenção da cegueira. Arq Bras Oftalmol 50: 145, 1987.

40 - COSTA VP; VASCONCELOS IPC; PELEGRINO M \& KARAJOSÉ N. O que os pacientes sabem sobre glaucoma? Arq Bras Oftalmol 58: 36-41, 1995.

41 - WENSOR MD; MCCARTY CA; STANISLAVSKY YL \& LIVINGSTON PM \& TAYLOR HR. The prevalence of glaucoma in the Melbourne visual impairment project. Ophthalmology 105: 733-737, 1998.

42 - KARA-JOSÉ N; CONTRERAS F; CAMPOS M; DELGADO A MOWERY R \& ELLWELN LB. Estudo populacional de triagem visual e intervenção cirúrgica para reduzir a cegueira por catarata em Campinas-Brasil e Chimbote-Peru. Ãrq Bras Oftalmol 52: 9I-99, 1989.
43 - KASS AM. Standardizing the measurement of intraocular pressure for clinical research. Ophthalmology 103: 183185, 1996.

44 - JOHNSON CA. Standardizing the measurement of visual fields for clinical research. Ophthalmology 103: 186-189, 1996.

45 - RODRIGUES MLV. Prevenção da cegueira. In: RODRIGLJES MLV. Oftalmologia clínica. Cultura Médica, Rio de Janeiro, cap.27, p.502-522, 1992.

46 - QUIGLEY HÁ; WEST SK; MUNHOZ B; MMBAGA BBO \& GLOVINSKY Y. Examination methods for glaucoma prevalence surveys. Arch Ophthalmol 111: 1409-141515, 1993.

47 - MUNDORF TK; ZIMMERMAN TJ; NARDIN GF \& KENDALL KS. Automated perimetry, tomometry and questionnaire in glaucoma screening. Am J Ophthalmol 108: 505-508, 1989.

48 - SHERWOOD ME; GARCIA-SIEKAVIZZA A; MELTZER MI; HEBERT A; BURNS AF \& MCGORRAY S. Glaucoma's impact on quality of life and its relation to clinical indicators. Ophthalmology 105: 561-566, 1998.

49 - TEMPORINI ER. Promoção da saúde ocular. Arq Bras Oftalmol 62: 82-84, 1999

50 - JAVITT JC. Preventig blindness in Americans: the need for eye health education. Surv Ophthalmol 40: 41-44, 1995.

51- TEMPORINI ER \& KARA-JOSÉ N. Níveis de prevenção de problemas oftalmológicos: propostas de investigação. Arq Bras Oftalmal 58: 189-192, 1995.

Recebido para publicação em 22/07/2002

Aprovado para publicação em 27/12/2002 